\title{
Multifocal primary cutaneous extranodal NK/T lymphoma nasal type*
}

\author{
Pedro de Vasconcelos ${ }^{1}$ \\ Luís Soares-Almeida ${ }^{1}$
}

\author{
Cristina Ferreira ${ }^{1}$ \\ Paulo Filipe ${ }^{1}$
}

DOI: http:/ /dx.doi.org/10.1590/abd1806-4841.20164090

\begin{abstract}
Nasal type extranodal NK/T-cell lymphoma is a distinct entity according to the World Health Organization classification. Although $60 \%$ to $90 \%$ of patients with this disease present with a destructive mass in the midline facial tissues, it may also primarily or secondarily involve extranasal sites, like the skin. We report the case of a 77-year-old patient that came to our department with erythematous plaques of the right leg and eczematous lesions of the trunk. These lesions were biopsied and the patient was diagnosed with extranodal NK/T-cell lymphoma, nasal type. He was treated with multi-agent systemic chemotherapy but died 5 months after diagnosis. This case highlights the rarity and variability of cutaneous features of this disease and its aggressive course and poor prognosis.
\end{abstract}

Keywords: Antineoplastic combined chemotherapy protocols; Lymphoma, extranodal NK-T-Cell; Skin

\section{INTRODUCTION}

Extranodal NK/T-cell lymphoma (ENKTL), nasal type, is commonly located in the upper respiratory tract, but involvement of other organs can be observed, particularly the skin, which is the second most usual site of this lymphoma after the nasal cavity and nasopharynx. Cutaneous ENKTL can be divided into two distinct subsets: primary cutaneous, that initially presents in the skin, or nasal ENKTL accompanied by secondary spread to the skin. ${ }^{1}$ Both forms are rare and are more prevalent in Asia, Central and South America. ${ }^{2}$ Epstein-Barr virus (EBV) is expressed in almost all cases of ENKTL, implying that it may play a pathogenic role. ${ }^{3}$ Extra-nasal ENKTL was first described in 1992 by Chan et al and this entity demonstrates many morphologic and immunophenotypic similarities to nasal ENKTL, thus the common designation "nasal-type" even for the extra-nasal forms.

\section{CASE REPORT}

A 77-year-old Caucasian man came to our outpatient clinic with large erythematous desquamative, very infiltrated, plaques on his right leg, with 4 months of evolution and no itching or other local or systemic symptoms (Figure 1). The patient informed that he had not been recently medicated with new drugs. According to his past medical history, he had diagnosis of diabetes mellitus type 2, 20 years ago, controlled with metformin and gliclazide. Two months after the onset of this condition, when plaques were smaller and less infiltrated, he was diagnosed with nummular eczema and treated with betamethasone dipropionate ointment, presenting no improvement.

On his lateral right abdomen there were also two small eczematous plaques, with no associated symptoms (Figure 2). The remainder physical examination was unremarkable and there were no palpable cervical, axillary, or inguinal lymph nodes. Laboratory

Received on 04.10 .2014

Approved by the Advisory Board and accepted for publication on 04.11.2014

Study performed at Hospital de Santa Maria, Serviço de Dermatologia - Lisboa, Portugal.

Financial Support: None.

Conflict of Interest: None.

Hospital de Santa Maria - Lisboa, Portugal.

C2016 by Anais Brasileiros de Dermatologia 


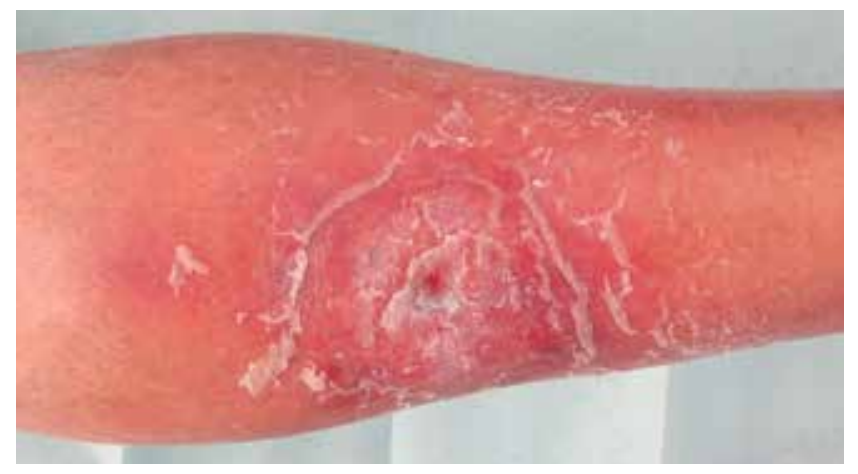

Figure 1: Extranodal NK/T lymphoma, nasal type: erythematous and desquamating, very infiltrated, plaques, some with central ulceration, on the right leg of a 77-year-old Caucasian man

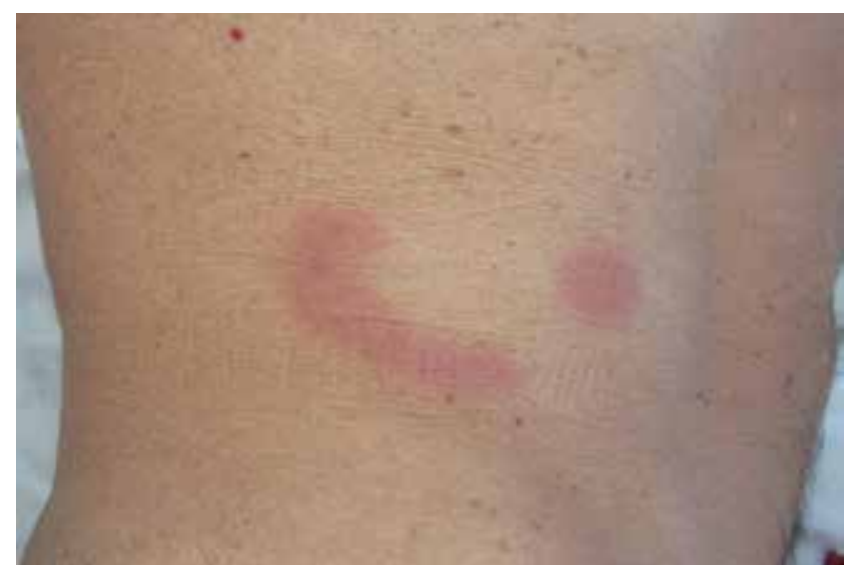

Figure 2: Eczematous lesions of the trunk with fine loose scaling

investigations revealed normal total white cell, lymphocyte, platelet and erythrocyte count and normal hemoglobin level. Erythrocyte sedimentation rate was $22 \mathrm{~mm} / \mathrm{h}$ and $\beta-2$ microglobulin, LDH and other biochemical parameters were within normal range.

A skin incisional biopsy was performed and its histopathological examination revealed dense dermal and hypodermal infiltration with small and medium-sized lymphocytes, with numerous mitosis, angiocentricity and epidermotropism (Figures 3 and 4). The immunohistochemical study showed positivity to $\mathrm{CD} 2$, CD3, CD56, granzime-B and TIA-1, and negativity to CD20, CD8 and CD30 (Figure 5). The proliferative index (ki-67) was very high $(80 \%)$ and EBV was demonstrated by in situ hybridization (Figure 6). Skin biopsy of a trunk lesion gave similar conventional histology and immunohistochemical results. These findings led us to a diagnosis of NK/T-cell lymphoma. Further investigations, including computed tomography of the thorax, abdomen and pelvis, bone marrow examination and nasoendoscopy gave no positive results. Thus the final diagnosis of primary cutaneous ENKTL, nasal type, was made.

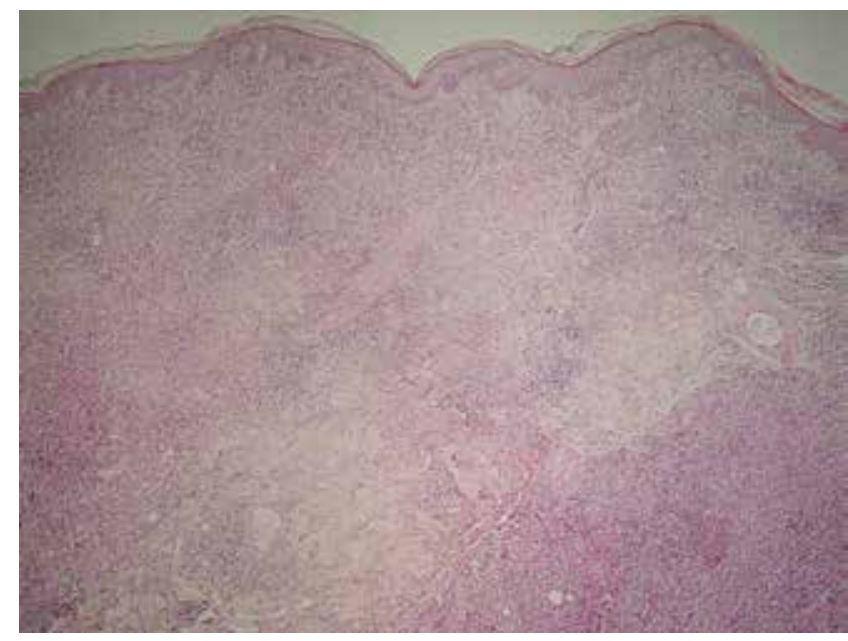

FIGURE 3: Diffuse proliferation of lymphocytes involving the dermis and subcutaneous tissues. Epidermotropism and angioinvasion is present $(\mathrm{H} \& \mathrm{E}$, original magnification $\times 40)$

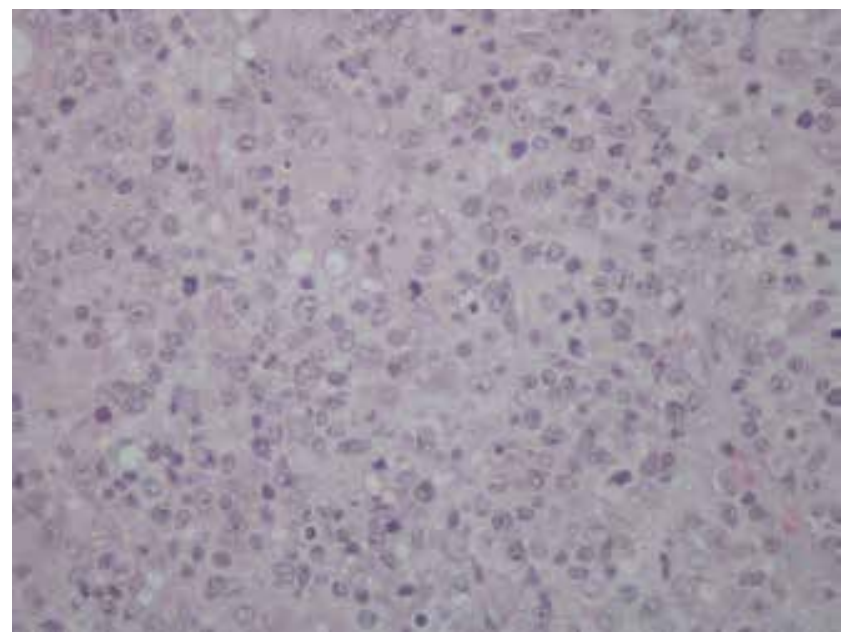

FIGURE 4: Dense deep infiltrate of the dermis with small, medium-sized and some large pleomorphic lymphocytes. Numerous mitosis are also seen (H\&E, original magnification $\times 400$ )

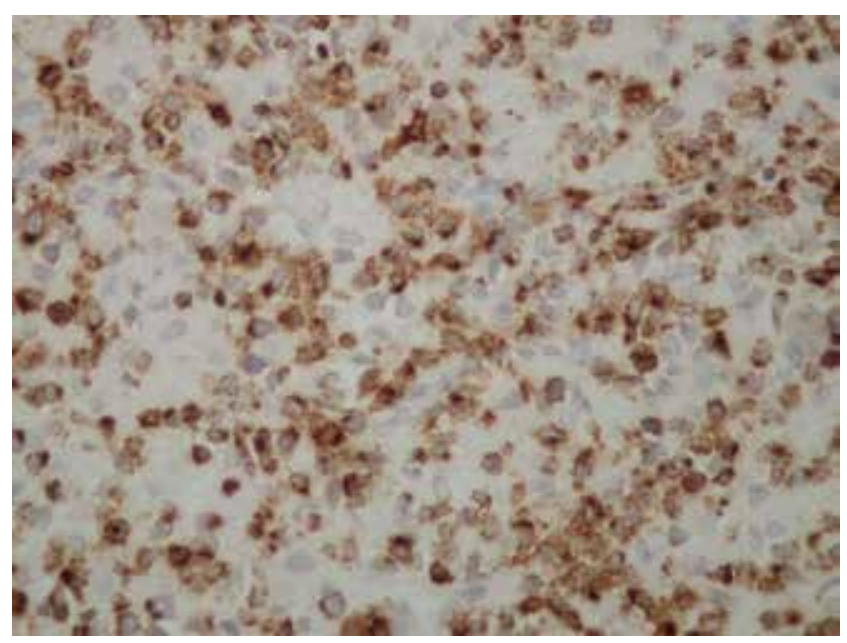

FIGURE 5: Positive granzyme B immunostaining (original magnification $x 400$ ). Granzyme B is a cytotoxic protein which is positive in almost all cases of extranodal NK/T lymphoma nasal type 


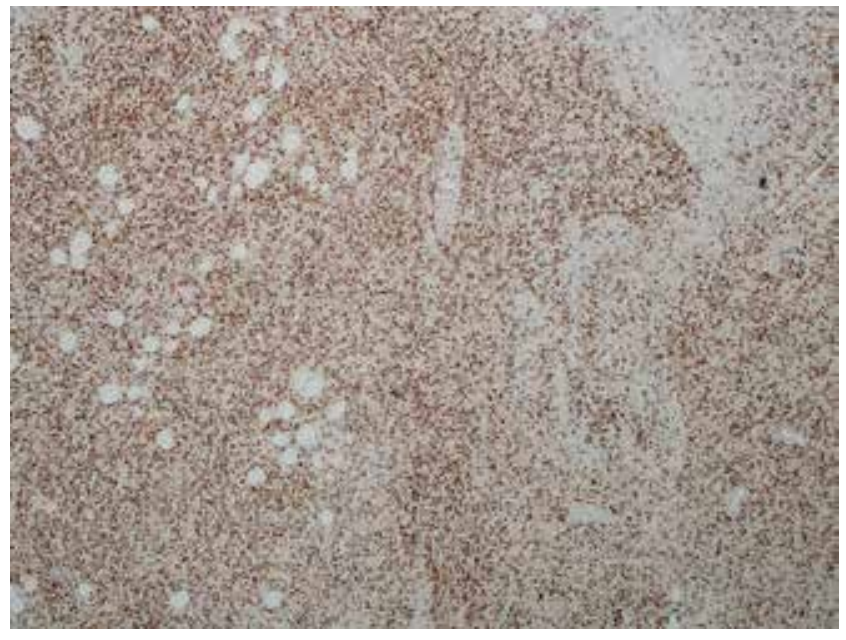

FIGURE 6: Intense positive ki-67 immunostaining indicating a proliferative index of $80 \%$

The patient was treated with CHOP chemotherapy regimen (intravenous infusion of cyclophosphamide $750 \mathrm{mg} / \mathrm{m}^{2}$, doxorubicin $50 \mathrm{mg} / \mathrm{m}^{2}$ and vincristine $2 \mathrm{mg} / \mathrm{m}^{2}$ once every 4 weeks and oral prednisolone $40 \mathrm{mg} / \mathrm{m}^{2}$ for 5 days every 4 weeks) with good initial response, with regression of plaques both in right leg and trunk. After 4 cycles of CHOP chemotherapy, 5 months after the diagnosis of ENKTL, the patient died from sepsis related complications.

\section{DISCUSSION}

Primary cutaneous ENKTL nasal type patients are usually adults, with a predominance of males. ${ }^{4}$ Clinical features are variable, but the most frequently observed skin lesions are erythematous or violaceous plaques and tumors, which are sometimes ulcerated. ${ }^{2}$ Upper respiratory tract should be checked at presentation and during follow-up, as involvement of this region is common. In some cases of ENKTL, nasal type, cutaneous features can be similar to those of mycosis fungoides.

As for many aggressive cutaneous cytotoxic lymphomas, overlapping clinicopathological features are common and classification can be difficult. ${ }^{5}$ Most cases have NK immunophenotype and are associated with EBV infection, with negativity for T-cell markers and germline rearrangement of T-cell receptor. ${ }^{6} \mathrm{Al}-$ though CD3 is negative in most cases, immunostainings can be positive to this marker due to the fact that the $\varepsilon$ chain of the CD3 molecule is usually expressed intracytoplasmically.

The treatment of choice is systemic chemotherapy, even in cases with involvement limited to the skin. ${ }^{6,7}$ However, ENKTL is very aggressive and generally fail to respond to multi-agent chemotherapy.

The prognosis of ENKTL, nasal type, is very poor and most patients die a few months after the diagnosis, being the estimated 5-year survival of $0 \%$. A better prognosis has been described in patients who have primary skin involvement, as opposed to those of nasal lymphoma and secondary skin manifestations. ${ }^{1}$

\section{REFERENCES}

1. Lee WJ, Jung JM, Won CH, Chang SE, Choi JH, Chan Moon K,et al. Cutaneous extranodal natural killer/T-cell lymphoma: a comparative clinicohistopathologic and survival outcome analysis of 45 cases according to the primary tumor site. J Am Acad Dermatol. 2014;70:1002-9

2. Chia HY, Tey HL, Tan KB, Chong WS. Nasal-type extranodal natural killer/T-cell lymphoma presenting with extensive leg ulcers. Clin Exp Dermatol. 2009;34:e693-5.

3. Hamada T, Nakamura S, Ko YH, Yoshino T, Ohshima K, Matsuzawa T, et al. Epstein-Barr virus-associated T/natural killer-cell lymphomas in the elderly: the first consensus meeting in Kofu 2013. J Dermatol. 2014;41:40-2.

4. Wang P, Sun Y, Wang Z, Xu A. Primary cutaneous extranodal NK/T-cell lymphoma, nasal type, in an adolescent. Eur J Dermatol. 2010;20:130-1.

5. Zuriel D, Fink-Puches R, Cerroni L. A case of primary cutaneous extranodal natural killer/t-cell lymphoma, nasal type, with a 22-year indolent clinical course. Am J Dermatopathol. 2012;34:194-7.

6. Stokkermans-Dubois J, Jouary T, Vergier B, Delaunay MM, Taieb A. A case of primary cutaneous nasal type NK/T-cell lymphoma and review of the literature. Dermatology. 2006;213:345-9.

7. Dearden CE, Johnson R, Pettengell R, Devereux S, Cwynarski K, Whittaker S, et al. Guidelines for the management of mature T-cell and NK-cell neoplasms (excluding cutaneous T-cell lymphoma). Br J Haematol. 2011;153:451-85.

\author{
MAILING ADDRESS: \\ Pedro de Vasconcelos \\ Hospital de Santa Maria \\ Serviço de Dermatologia \\ Avenida Professor Egas Moniz, \\ 1649-035 Lisboa \\ Email:jp_vasconcelos@hotmail.com
}

How to cite this article:de Vasconcelos P, Ferreira C, Soares-Almeida L, Filipe P. Multifocal primary cutaneous extranodal NK/T lymphoma nasal type An Bras Dermatol. 2016;91(2):219-21. 